\title{
The Effect of Fasting on Rat Portal Venous and Aortic Blood Glucose, Lactate, Alanine, and Glutamine
}

\author{
ROBERT E. KIMURA, TIMOTHY R. LAPINE, JERRILYN JOHNSTON, AND JASMINKA Z. ILICH \\ Department of Pediatrics, Division of Neonatology, University of Utah School of Medicine, \\ Salt Lake City, Utah 84132
}

\begin{abstract}
Using a chronically catheterized rat model, the effect of fasting on portal venous, aortic, and venous blood concentration minus aortic blood concentration ([PVA]) blood glucose, lactate, alanine, and glutamine concentrations was determined. It has been postulated that the intestine is a source of lactate and alanine, precursors for glycogen synthesis, in the fed state. After $48 \mathrm{~h}$ of fasting portal venous glucose, lactate, and alanine blood concentrations decreased by 31,28 , and $41 \%$, respectively. Portal venous glutamine concentration was not affected by fasting. $A$ glucose [PV-A] was not found in either fed or fasted states. Whereas the lactate $[\mathrm{PV}-\mathrm{A}]$ was not present in fed rats, it was negative in fasted rats. Alanine [PV-A] was positive in fed and fasted rats. The glutamine [PV-A] was negative in fed and fasted rats. These data indicate that portal venous concentrations of the gluconeogenic precursors, lactate and alanine, decrease in fasted rats. In fasted rats intestinal utilization of lactate increases as reflected by a negative $[\mathrm{PV}-\mathrm{A}]$. Fasting did not affect alanine production by the intestine or glutamine utilization. Despite these changes with fasting, we conclude that the intestine does not appear to be able to maintain portal venous blood concentrations of gluconeogenic precursors. (Pediatr Res 23: 241-244, 1988)
\end{abstract}

Abbreviation

[PV-A], portal venous blood concentration minus aortic blood concentration

Many low birth weight infants are nourished using parenteral methods. If the intestine is primarily an absorptive organ, this therapy may be appropriate. However, the small intestine performs several major metabolic functions including oxidation of glucose to lactate under aerobic conditions (1-3), oxidation of glutamine to $\mathrm{CO}_{2}(3,4)$, and conversion of lactate to alanine and $\mathrm{CO}_{2}$ after infusions of lactate (3). These important intestinal metabolic functions release gluconeogenic precursors, alanine and lactate, into the portal venous effluent which perfuses the liver. Ramesy et al. (5) have reported elevated portal venous concentrations of glucose, alanine, and lactate in fed rats. Katz et al. (6) and Boyd et al. (7) have reported that gluconeogenic precursors, alanine (8) and lactate, are the primary precursors for hepatic glycogen synthesis during the fed state. Together,

Received July 24, 1987; accepted October 14, 1987.

Correspondence Dr. R. E. Kimura, University of Utah Medical School, Division of Neonatology, 2A210, Department of Pediatrics, 50 North Medical Drive, Sal Lake City, UT 84132.

Supported in part by NIH Grant 2 RO1 HD 18517-04. these data suggest that the small intestine may be a significant source of lactate and alanine for utilization by the liver. During fasting, intestinal glutamine oxidation to $\mathrm{CO}_{2}$ and the subsequent production of alanine are not affected $(3,9)$ and so the intestine may be an important source of alanine.

These studies, which identified significant aerobic small intestinal metabolism of glucose to lactate and the oxidation of glutamine to $\mathrm{CO}_{2}(1-4)$, were done under anesthesia and with bowel manipulation. Anesthesia can alter blood substrate concentrations as well as the metabolism of substrates (3). Ether, for example, increases blood glucose and lactate concentrations (10). Anesthesia also can decrease cardiac output which would decrease mesenteric blood flow and intestinal perfusion. Surgical manipulation can alter intestinal metabolism and perfusion. Metabolic studies involving the fetal lamb exemplify the importance of chronic catheterization in contrast to acute catheterization (11).

We set out to determine the effect of fasting on small intestinal metabolism and the resulting effect on portal venous blood substrate concentrations under physiologic conditions. We used a chronically catheterized rat model in which the portal vein and aorta remained patent for blood sampling. The portal venous and aortic blood concentrations of glucose, lactate, alanine, and glutamine were measured in the fasted and fed states. In the fasting state intraluminal substrates are unavailable to the intestine, so the portal venous to aortic blood concentration gradient reflects qualitatively the utilization or production of blood borne substrates by the intestine.

\section{MATERIALS AND METHODS}

Animals. All experiments were performed in vivo using adult male, albino, Sprague-Dawley rats $(200-300 \mathrm{~g}$ body weight). These animals were obtained from Simonsen Labs (Gilroy, CA) and allowed free access to water and rat food. The adult rats were weighed and anesthetized with a $20 \mathrm{mg} / \mathrm{kg}$ ketamine injected intramuscularly followed by a $10 \mathrm{mg} / \mathrm{kg}$ pentobarbital injected intraperitoneally. Using aseptic technique, portal venous and aortic catheters were surgically placed as described previously (12). After surgery, animals were weighed daily and the catheters were flushed with $0.35 \mathrm{ml}$ of normal saline containing $500 \mathrm{U} /$ $\mathrm{ml}$ heparin and $2.5 \mathrm{mg} / \mathrm{ml}$ of ampicillin.

The effect of fasting on portal venous and aortic blood substrate concentrations. The effects of fasting on portal venous and aortic blood substrate concentrations were investigated in rats who were catheterized for more than 4 days. Portal venous and aortic blood $(0.4 \mathrm{ml})$ samples were drawn and immediately placed in $0.4 \mathrm{ml}$ of ice cold $1 \mathrm{M} \mathrm{HClO}_{4}$. The protein precipitate was separated from the remaining solution by centrifugation and the supernatant was neutralized with $2 \mathrm{M}$ potassium bicarbonate within $60 \mathrm{~min}$ of blood sampling. To determine blood substrate 
concentrations in small volumes of blood, we adapted spectrophotometric assays for glucose (13), lactate (14), alanine (15), and glutamine (16), to fluorometric assays. Blood samples were analyzed for substrate concentrations within $2 \mathrm{~h}$. Because Aoki et al. (17) reported that red blood cells transport significant amounts of amino acids, we measured whole blood and not plasma concentrations of all substrates in order to determine portal venous and aortic blood concentrations differences ([PVA]) as an indication of net flux of substrates across the gastrointestinal tract. A positive [PV-A] implies substrate production by the intestine.

Units for substrate concentrations are umol of substrate/g blood (mean \pm SEM). Statistical significance was evaluated using the unpaired Student's $t$ test with the Bonferroni correction for multiple $t$ tests.

\section{RESULTS}

The chronically catheterized rat preparation. Three days after placement of the catheters, the rats had lost $10 \%$ of their preoperative weight. The weights remained constant for the next 4 days and then increased $1.4 \%$ of preoperative weight per day thereafter. Preoperative weight was regained $10.8 \pm 0.7$ days after surgery. Fifteen experiments were carried out in rats 4-9 days after catheter placement and 13 experiments were carried out in rats more than 10 days after catheter placement.

The effect of fasting on aortic and portal venous substrate concentrations in chronically catheterized rats. Glucose and Lactate. After $48 \mathrm{~h}$ of fasting, mean glucose concentrations decreased in portal venous and aortic blood by 32 and $26 \%$, respectively $(p<0.001)$ (Fig. 1). A significant glucose [PV-A] was not found in fed or fasted catheterized rats.

Aortic lactate concentrations in catheterized rats did not change significantly after $48 \mathrm{~h}$ of fasting (Fig. 1). Mean portal venous lactate concentrations decreased from fed concentrations by $46 \%(p<0.001)$. A significant lactate [PV-A] was not present in fed rats. The lactate [PV-A] of fasted rats was significantly negative (paired $t$ test, $p<0.012$ ).
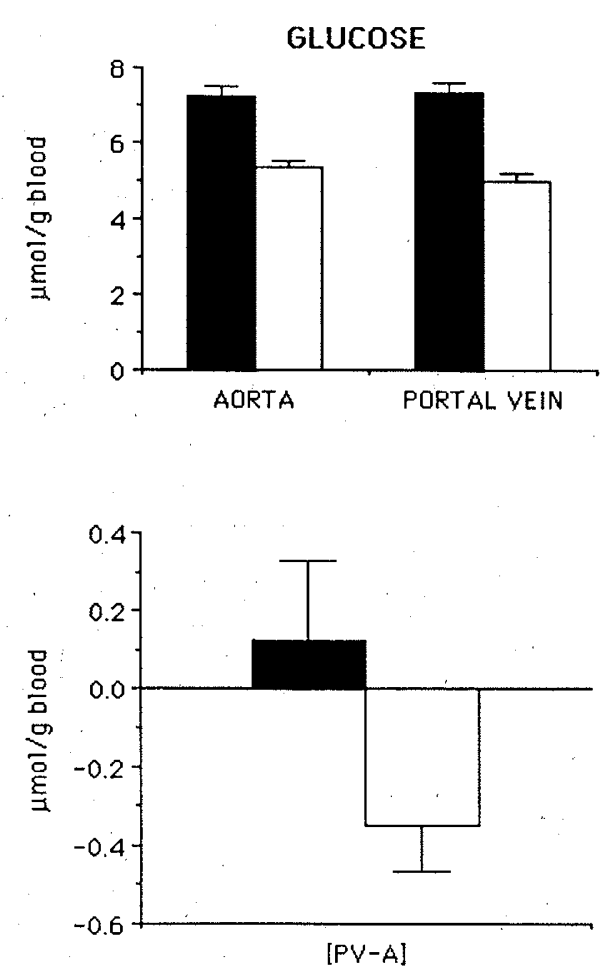

Fig. 1. Aortic and portal venous blood glucose and lactate concentrations were determined in the fed state ( $\square$ ) and after $48 \mathrm{~h}$ of fasting ( $\square$ ). The values are means $\pm \operatorname{SEM}(n=11)$.

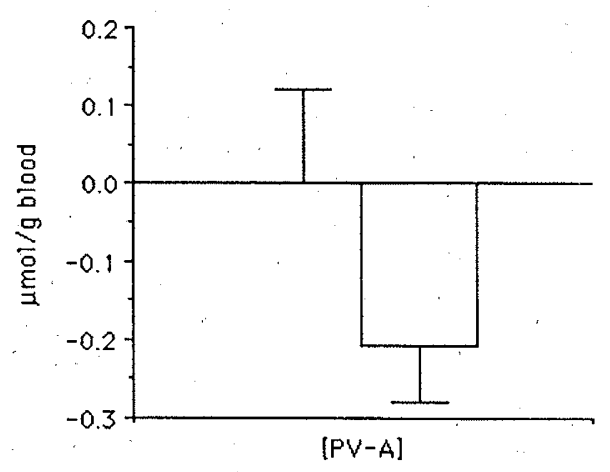

Alanine. Mean aortic alanine and portal venous alanine concentrations of fasted catheterized rats were $42 \%(p<0.005)$ and $47 \%(p<0.001)$ less than concentrations in the fed state, respectively. The alanine [PV-A] was positive (paired $t$ test, $p<$ 0.005 ) in fed rats (Fig. 2). In fasted rats the alanine [PV-A] remained positive (paired $t$ test, $p<0.001$ ) but was one-half that in the fed state.

Glutamine. No significant change in mean aortic or portal venous glutamine concentrations were found between the fasted and fed states in chronically catheterized rats. Glutamine [PVA] was negative in fed (paired $t$ test, $p<0.005$ ) and fasted (paired $t$ test, $p<0.001$ ) rats (Fig. 2). Fasting for $48 \mathrm{~h}$ did not alter the magnitude of the glutamine [PV-A].

\section{DISCUSSION}

During the fed state, the glucose [PV-A] in chronically catheterized rats varied from animal to animal. Because these animals were allowed unrestricted access to food, the variability in the glucose [PV-A] may reflect different amounts of rat food present in the intestine at the time of the study. In fed catheterized rats the portal venous lactate concentrations were not significantly greater than aortic concentrations indicating no significant lactate production by the intestine. This in vivo finding in a unanesthetized rat is consistant with data of Windmueller and Spaeth (3) who used an anesthetized in situ intestinal preparation. In contrast, Nicholls et al. (2) using a perfused intestinal model measured a significant amount of lactate production. Hanson and Parsons (1) determined that the amount of intestinal lactate production in a perfused model was dependent on the hematocrit of the perfusate. These studies indicate that presumed aerobic lactate production by intestine may actually be an artifact of the intestinal model. In our in vivo model without anesthesia, no significant lactate production was determined.

We found in our study that aortic glucose and lactate concentrations decreased after $48 \mathrm{~h}$ of fasting. This observation is to a decrease in blood glucose and lactate concentrations asso-

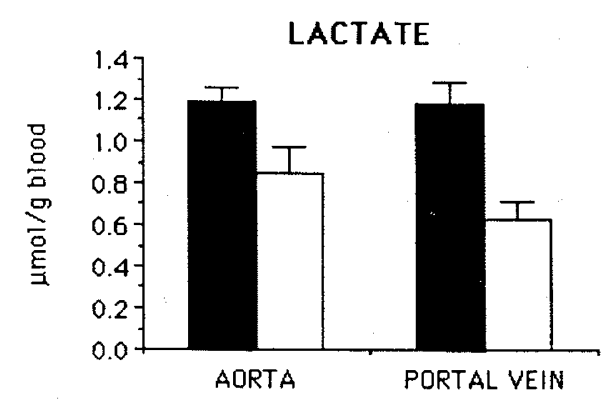
consistent with the data of Ballard and Hanson (18). In addition 

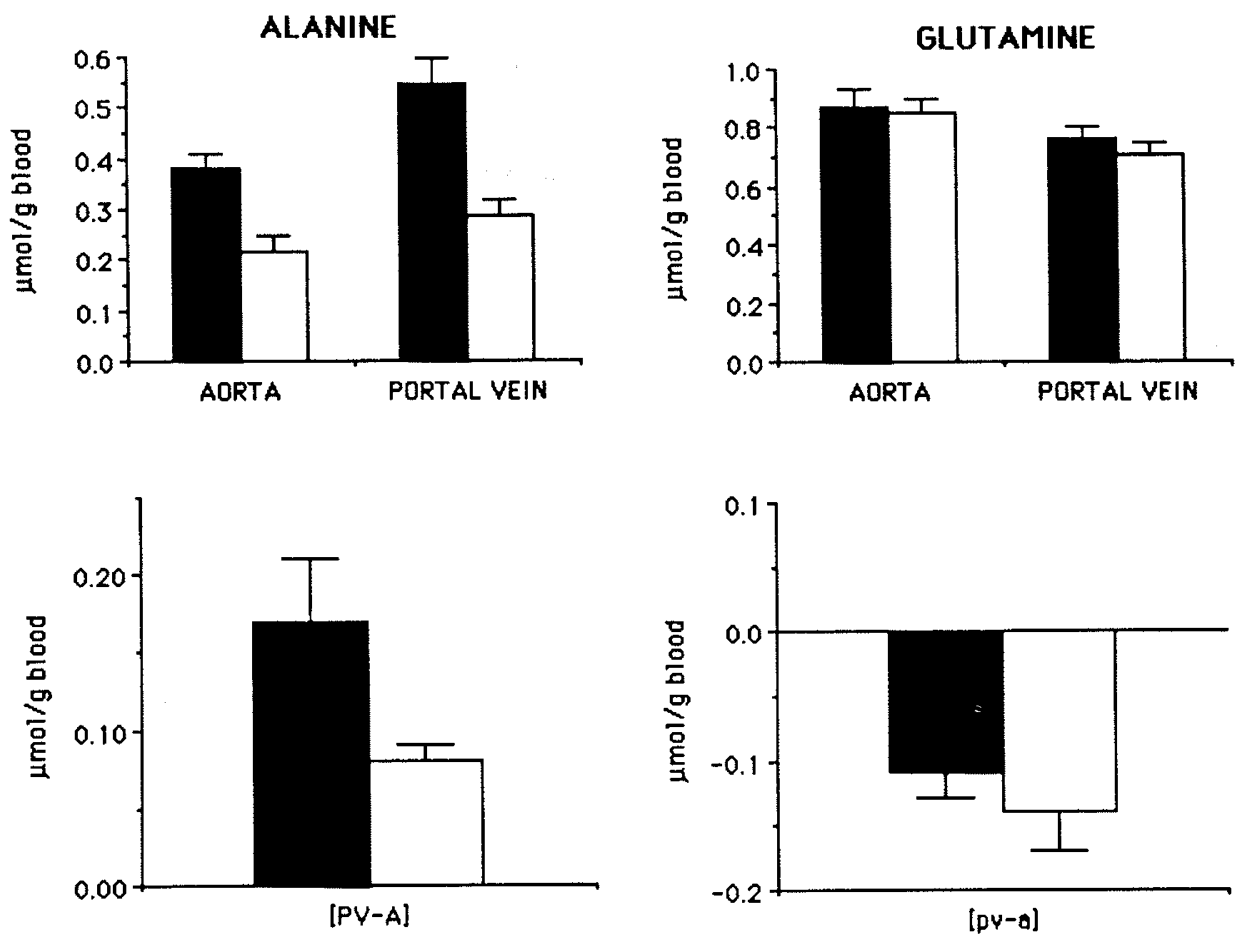

Fig. 2. Aortic and portal venous blood alanine and glutamine concentrations were determined in the fed state ( $\square$ ) and after $48 \mathrm{~h}$ of fasting ( $\square$ ). The values are means $\pm \operatorname{SEM}(n=8-11)$.

ciated with fasting, the lactate [PV-A] was negative after $48 \mathrm{~h}$ of fasting in chronically catheterized rats, suggesting active uptake and utilization of lactate by the intestine. Because intraluminal substrates are low in the fasted state, the negative lactate [PV-A] reflects intestinal utilization of vascular borne lactate. Most probably lactate is converted to pyruvate which is then converted to alanine by alanine aminotransferase. Windmueller and Spaeth (3) determined that $60 \%$ of lactate infused into the mesenteric vascular system was converted to alanine. The conversion of lactate to alanine, is supported by the observation that there is a positive alanine [PV-A] in the fasted rat at $48 \mathrm{~h}$. It is possible that lactate can be used as an oxidative substrate by the small intestine. However, in experiments by Windmueller and Spaeth (3) and Hanson and Parsons (9), lactate does not appear to be a primary oxidative substrate in the postabsorptive intestine. Another possibility is the conversion of lactate to glucose through gluconeogenesis. However, Hahn and Wei-ning (19) have reported that gluconeogenesis in intestinal mucosa is low in adult rats.

Portal venous lactate concentrations decreased in chronically catheterized fasted rats. This decline in portal venous lactate concentration reflects the decrease in aortic lactate concentrations. In addition, in fasted chronically catheterized rats the lactate [PV-A] was negative suggesting lactate utilization by the small intestine, which would cause a decrease in portal venous concentration. These results suggest that in the fasting state there is a decrease availability of lactate as a precursor for gluconeogenesis.

It has been proposed that the small intestine is a significant source of alanine $(20,21)$. We measured a significantly positive alanine [PV-A] in both the fed and fasted states. The positive alanine [PV-A] in fasted rats must be secondary to net production of alanine by the small intestine and not related to absorption of intraluminal amino acids. The production of alanine from lactate infused into an intestinal artery has been reported by Windmueller and Spaeth (3). The significantly greater alanine [PV-A] in fed compared to fasted chronically catheterized rats may be secondary to the intraluminal source of alanine available in the fed state. This is supported by the decrease in PV alanine in fasted chronically catheterized rats compared to fed rats. The actual rate of production of alanine by the intestine has not been determined because we have not measured the rate of mesenteric blood flow.

Glutamine oxidation by the intestine involves the conversion of pyruvate to alanine via alanine aminotransferase and the conversion of glutamate to 2-oxoglutarate. We have determined that in both the fed and fasted states the glutamine [PV-A] is negative indicating a net utilization of glutamine by the small intestine. Our observations that fasting does not affect glutamine utilization by the intestine as indicated by the glutamine [PV-A] is consistent with the results reported by Hanson and Parsons (8) and Windmueller and Spaeth (3). It has been reported that glutaminase activity in rat mucosa is not affected by fasting (22, 23). The actual rate of utilization of glutamine has not been determined because we have not determined the rate of mesenteric blood flow.

In in vitro experiments, lactate, alanine, and galactose, gluconeogenic precursors, were the primary source of carbon in glycogen synthesis. These studies indicated that glucose is not incorporated into liver glycogen (5). In elegant studies, Newgard et al. (24) showed that in order for glucose carbon to be incorporated into liver glycogen, it must first be metabolized to pyruvate and then synthesized to glucose-6-phosphate by gluconeogenesis before glycogen synthesis. The intestine has been implicated as a significant organ for the production of lactate and alanine (7). Our study indicates that the intestine does produce alanine. However, we were not able to measure a positive lactate [PV-A] in either the fed or fasting states.

In summary, these in vivo studies in unanesthetized rat indicate that the small intestine is a source of alanine and a utilizer of glutamine. In addition, these studies indicate that gluconeogenic precursors, lactate and alanine, decrease in the portal vein with fasting. It is known that aerobic metabolism of glucose to lactate occurs in the fasting state. However, the lactate [PV-A] is negative in the fasting state and this suggests net intestinal utilization. The alanine [PV-A] remain positive during the fasting state suggesting intestinal production of alanine. Despite these changes with fasting, we conclude that the intestine does not appear to be able 
to maintain portal venous blood concentrations of gluconeogenic precursors.

\section{REFERENCES}

1. Hanson PJ, Parsons DS 1976 The utilization of glucose and production of lactate by in vitro preparations of rat small intestine: effects of vascular perfusion. J Physiol 255:775-795

2. Nicholls TJ, Leese HJ, Bronk JR 1983 Transport and metabolism of glucose by rat small intestine. Biochem J 212:183-187

3. Windmueller HG, Spaeth AE 1978 Identification of ketone bodies and glutamine as the major respiratory fuels in vivo for postabsorptive rat small intestine. J Biol Chem 253:69-76

4. Windmueller HG, Spaeth AE 1980 Respiratory fuels and nitrogen metabolism in vivo in small intestine of fed rats. J Biol Chem 255:107-112

5. Remesy C, Demigne C, Aufrere J 1978 Inter-organ relationships between glucose, lactate and amino acids in rats fed on high-carbohydrate or highprotein diets. Biochem J 170:321-329

6. Katz J, Golden S, Wals PA 1979 Glycogen synthesis by rat hepatocytes. Biochem J 180:389-402

7. Boyd M, Albright E, Roster D, McGarry J 1981 In-vitro reversal of the fasting state of liver metabolism in the rat. J Clin Invest 68:142-152

8. Ishikawa E, Aikawa T, Matsutaka H 1972 The roles of alanine as a major precursor among amino acids of hepatic gluconeogenesis and as a major end product of the degradation of amino acids in rat tissues. J Biochem 71:10971099

9. Hanson PJ, Parsons DS 1978 Factors affecting the utilization of ketone bodies and other substrates by rat jejunum: effects of fasting and of diabetes. $\mathbf{J}$ Physiol 278:55-67

10. Brewster WR Jr, Bunker JP, Beecher HK 1952 Metabolic effect of anesthesia. VI. Mechanism of metabolic acidosis and hyperglycemia during ether anesthesia in the dog. Am J Physiol 1781:37-47

11. Meschia G, Makowshi EL, Battaglia FC 1970 The use of indwelling catheter in the uterine and umbilical veins of sheep for a description of fetal acid-gas balance and oxygenation. Yale J Biol Med 42:154-165
12. Kimura RE, LaPine TR, Gooch WM III 1988 Portal venous and aortic glucose and lactate changes in a chronically catheterized rat. Pediatr Res 23:235240

13. Shein MW 1965 D-Glucose: determination with hexokinase and glucose-6 phosphate dehydrogenase. In: Bergmeyer HU (ed) Methods of Enzymatic Analysis. Academic Press, New York, pp 117-123

14. Hohorst H-J 1965 L-(+)-Lactate determination with lactate dehydrogenase and DPN. In: Bergmeyer HU (ed) Methods of Enyzmatic Analysis. Academic Press, New York, pp 846

15. Williamson DH 1974 L-alanine determination with alanine dehydrogenase. In: Bergmeyer HU (ed) Methods of Enzymatic Analysis, Vol 4. Academic Press, New York, pp 1679-1685

16. Lund $P 1974$ Determination with glutaminase and glutamate dehydrogenase. In: Bergmeyer HU (ed) Methods of Enzymatic Analysis, Vol 4. Academic Press, New York, pp 1719-1722

17. Aoki TT, Brennan MF, Muller WA, Cahill GF Jr 1974 Amino acid levels across normal forearm muscle: whole blood vs. plasma. Adv Enzyme Regul 12:157-165

18. Ballard FJ, Hanson RW 1969 Measurement of adipose-tissue metabolites in vivo. Biochem J 112:1956-202

19. Hahn P, Wei-ning H 1986 Gluconeogenesis from lactate in the small intestinal mucosa of suckling rats. Pediatr Res 20:1321-1323

20. Bradford NM, McGiven JD 1982 The transport of alanine and glutamine into isolated rat intestinal epithelial cells. Biochem Biophys Acta 689:55-62

21. Neame KD, Wiseman G 1958 The alanine and oxo acid concentrations in mesenteric blood during the absorption of $\mathrm{L}$-glutamic acid by the small intestine of the dog, cat and rabbit in vivo. J Physiol 140:148-155

22. Budohoski L, Challis RAJ, Newsholme EA 1982 Effects of starvation on the maximal activities of some glycolytic and citric acid cycle enzymes and glutaminase in mucosa of the small intestine of the rat. Biochem J 206:169172

23. McFarlane Anderson N, Bennett FI, Alleyne GAO 1976 Ammonia production by the small intestine of the rat. Biochem Biophys Acta 437:238-243

24. Newgard CB, Hirsch LJ, Foster DW, McGarry JD 1983 Studies on the mechanism by which exogenous glucose is converted into liver glycogen in the rat. J Biol Chem 258:8046-52 